\title{
Calcifediol treatment and COVID-19-related outcomes
}

Xavier Nogues ${ }^{1,2}$, Diana Ovejero ${ }^{1,2}$, Marta Pineda-Moncusí ${ }^{1}$, Roger Bouillon ${ }^{3}$, Dolors Arenas ${ }^{4}$, Julio Pascual $^{4}$, Anna Ribes ${ }^{1}$, Robert Guerri-Fernandez ${ }^{1,5}$, Judit Villar-Garcia ${ }^{5}$, Abora Rial ${ }^{2}$, Carme Gimenez-Argente ${ }^{2}$, Maria Lourdes Cos $^{2}$, Jaime Rodriguez-Morera ${ }^{2}$, Isabel Campodarve ${ }^{2}$, José Manuel Quesada-Gomez ${ }^{6^{*}}$ and Natalia Garcia-Giralt ${ }^{*}$

*Both authors contributed equally to this work

1. IMIM (Hospital del Mar Medical Research Institute), Centro de Investigación Biomédica en Red de Fragilidad y Envejecimiento Saludable (CIBERFES), Barcelona, Spain.

2. Internal Medicine Department, Hospital del Mar, Universitat Autònoma de Barcelona, Barcelona, Spain.xnogues@parcdesalutmar.cat

3. Clinical and Experimental Endocrinology, Department of Chronic Diseases and Metabolism, KU Leuven, Herestraat, 3000 Leuven, Belgium. Roger.bouillon@kuleuven.be - orchid number 0000 0002-6446-6763

4. Department of Nephrology, Hospital del Mar-IMIM, Barcelona, Spain.

5. Department of Infectious Diseases, Hospital del Mar-IMIM, Barcelona, Spain. rguerri@imim.es

6. Instituto Maimónides de Investigación Biomédica de Córdoba (IMIBIC). Fundación Progreso y Salud. CIBER de Fragilidad y Envejecimiento Saludable (CIBERFES). Hospital Universitario Reina Sofía. Universidad de Córdoba. Menéndez Pidal s/n, 14004, Córdoba, Spain. imquesada@uco.es

Corresponding author:

Natalia Garcia-Giralt, $\mathrm{PhD}$

Postdoc Researcher in CIBER in frailty and healthy ageing

Associate Lecturer of University of Barcelona

e-mail:ngarcia@imim.es

ORCID:0000-0001-6507-0147

(C) The Author(s) 2021. Published by Oxford University Press on behalf of the Endocrine Society. All rights reserved. For permissions, please e-mail: journals.permissions@oup.com 
Disclosure Statement: XN has received fees from lectures and consultancy from Amgen, UCB, and Lilly. RB has received small lecture or consultancy fees from Fresenius (Germany), Abiogen (Italy), Faes Farma (Spain) and Proctor \& Gamble (Belgium). JMQG has received small lecture or consultancy fees from Amgen and Faes Farma (Spain). All other authors have nothing to declare. 


\section{ABSTRACT}

Context. COVID-19 is a major health problem because of saturation of intensive care units (ICU) and mortality. Vitamin D has emerged as a potential treatment able to reduce the disease severity.

Objective. To elucidate the effect of calcifediol [25OHD3] treatment on COVID-19-related outcomes.

Design. Observational cohort study from March to May, 2020.

Setting. Patients admitted to COVID-19 wards of Hospital del Mar, Barcelona, Spain.

Patients. A total of 930 patients with COVID-19 were included. Ninety-two were excluded due to previous calcifediol intake.

Intervention. Of the remaining 838 , a total of 447 received calcifediol (532ug on day one plus 266ug on day $3,7,15$, and 30 ) whereas 391 were not treated at the time of hospital admission (Intention-toTreat). Of the latter, 53 patients were treated later during ICU admission and were allocated in the treated group in a second analysis. In healthy subjects, calcifediol is about 3.2-fold more potent on a weight basis than cholecalciferol.

Main Outcome Measures. ICU admission and mortality.

Results. ICU assistance was required by 102 (12.2\%) participants. Out of 447 patients treated with calcifediol at admission, $20(4.5 \%)$ required ICU, compared to $82(21 \%)$ out of 391 non-treated ( $p$ value $<0.0001$ ). Logistic regression of calcifediol treatment on ICU admission, adjusted by age, gender, linearized 25OHD levels at baseline, and comorbidities showed that treated patients had a reduced risk to require ICU (OR $0.13[95 \% \mathrm{CI} 0.07 ; 0.23])$.

Overall mortality was $10 \%$. In the Intention-to-Treat analysis, $21(4.7 \%)$ out of 447 patients treated with calcifediol at admission died compared to 62 patients $(15.9 \%)$ out of 391 non-treated $(p=0.0001)$. 
Adjusted results showed a reduced mortality risk with an OR 0.21 [95\% $\mathrm{Cl} 0.10 ; 0.43])$. In the second analysis, the obtained OR was $0.52[95 \% \mathrm{Cl} 0.27 ; 0.99]$.

Conclusions. In patients hospitalized with COVID-19, calcifediol treatment significantly reduced ICU admission and mortality.

Key Words: COVID-19, Vitamin D, Calcifediol, ICU admission, mortality 
Abbreviations: COVID-19, coronavirus disease 2019; ICU, intensive care units; OR, Odds ratio; $\mathrm{Cl}$, Confidence interval; ITT, Intention-to-Treat; ARDS, acute respiratory distress syndrome; CURB65, disease severity scoring: the combination of Confusion, Urea, Respiratory rate, Blood pressure, and Age $\geq 65$; MEWS, Modified Early Warning Score consists of 5 physiological parameters, which include systolic blood pressure, pulse rate, respiratory rate, temperature, and level of consciousness. 


\section{Introduction}

Severe acute respiratory syndrome coronavirus (SARS-CoV-2) is the etiologic agent of Coronavirus disease-19 (COVID-19), a rapidly spreading infection that has caused a global pandemic since its initial identification in China in December 2019. The clinical spectrum of the disease ranges from asymptomatic or mild flu-like symptoms to severe respiratory illness and death (1). Epidemiological studies have estimated that approximately $20 \%$ of infected patients sought medical attention, and $10 \%$ required hospitalization, thereby tremendously impacting healthcare services worldwide $(2,3)$. The burden of COVID-19 has particularly overwhelmed critical care, leading to saturated intensive care units (ICU) by patients with SARS-CoV-2-mediated acute respiratory distress syndrome (ARDS). Therefore, there is an enormous interest by the medical and scientific community to identify risk and protective factors associated with the development of COVID-19-related severe outcomes. At the beginning of the pandemic no effective treatments were available. Even today only four drugs are approved by the FDA: the antiviral agent remdesivir, effective in reducing recovery time in adults hospitalized with COVID-19 (4) and dexamethasone, or other corticosteroids, the only therapy that has been shown to reduce mortality so far in patients requiring mechanical ventilation or high-flow oxygen (5). More recently, the FDA has approved by Emergency Use Authorization of baricitinib in combination with remdesivir in patients requiring invasive mechanical ventilation or extracorporeal membrane oxygenation (6), as well as the neutralizing antibody bamlanivimab (LY-CoV555) (7)

Adequate vitamin D status has emerged as a potentially preventing factor for COVID-19 progression and mortality $(8,9)$. Vitamin D endocrine system's (VDES) protective effects are supported by studies that point to a role of the hormone in innate and acquired immunity modulation, autophagy induction, and synthesis of reactive oxygen intermediates $(10,11)$. In addition, vitamin $D$ is known to be involved in the induction of antimicrobial peptides in response to both viral and bacterial infections (12). A systematic review and meta-analysis from randomized controlled trials including more than 11,000 participants before the onset of COVID-19, showed protective effects of daily or weekly vitamin $\mathrm{D}$ administration on the risk of acute respiratory tract infection (13). Many data indicate that vitamin D deficiency, which is common among critically ill patients, is associated with longer hospital and ICU length of stay, lung and other organ injury, prolonged mechanical ventilation, and death (14). 
Recent studies suggested that vitamin D supplementation might reduce the risk of influenza and SARS-CoV-2-related infections and deaths by decreasing pro-inflammatory cytokine production, thereby, diminishing the risk of developing a cytokine storm, which ultimately leads to ARDS development (15). A 2017 meta-analysis of data from 25 randomised controlled trials (RCTs) of vitamin D supplementation for the prevention of acute respiratory infections revealed a protective effect of this intervention, although the risk reduction was small. Protection was associated with administration of daily doses of $400-1000$ IU for up to 12 months (16).

Considering the aforementioned data, we evaluated the effect of calcifediol treatment and baseline 25OHD serum levels on COVID-19-related severe outcomes: ICU admission and mortality rate among patients hospitalized for COVID-19 in a population-based study on patients admitted to COVID-19 wards in Hospital del Mar, Barcelona, Spain.

\section{Methods}

\section{Study design}

Clinical data from patients with COVID-19, hospitalized randomly at one of the eight COVID-19 Units in Hospital del Mar (Barcelona, Spain), were collected from March to May 2020 in the BarnaCOVIDIOL cohort - a prospective, non-selected, observational, clinical cohort study. Each COVIDward from Hospital del Mar was assigned to one single Medical Unit during the first pandemic outbreak in Barcelona. The study protocol was approved by the ethics committee of Parc de Salut Mar (Exp number 2020/9287 and 2021/9751) and it was conducted in accordance with the Declaration of Helsinki and the Good Clinical Practice guidelines of the International Conference on Harmonization. All patients were verbally informed about the treatment options. Verbal consent was registered in the hospital's electronic medical record system. 


\section{Participants}

Patients with SARS-CoV-2 infection with chronic conditions and/or severe COVID-19 symptomatology were enrolled at admission. If patient met one of these conditions was admitted in one of the COVID19 wards:

In both options the SARS-CoV-2 PCR positive

1) Severe Pneumonia confirmed with $x$ Ray or CT scan CURB-65 $>2$

Oxygen saturation $<90 \%$

Respiratory rate $>30$

MEWS score 3-4

2) Mild Pneumonia confirmed with xRay or CT scan CURB-65 $\leq 1$

Oxygen saturation $\geq 90 \%$

Respiratory rate $\leq 30$

MEWS score $<3$

With chronic diseases as Chronic Obstructive Pulmonary Disease (COPD), Chronic Kidney Disease (CKD), Obesity, Diabetes, age $>65$

Patients were followed from the first day of admission to their COVID ward up to date of medical discharge or death. Eligible participants were patients aged 18 years or older who tested PCR positive for SARS-CoV-2. A total of 930 were included in the Barna-COVIDIOL cohort (Figure 1). Of them, 92 patients had been recently treated ( $<3$ months) with calcifediol and were excluded from the study. Finally, 838 patients were included in the Intention-to-Treat (ITT) analysis: 447 received calcifediol at admission to the hospital and 391 were not treated (control group). Fifty-three patients of the control group who required ICU also received calcifediol during ICU admission (same dosage and schedule as for patients treated at admission) at the discretion of the treating physicians and outside the original protocol.

Clinical samples for SARS-CoV-2 testing were obtained and analysed according to WHO guidelines [Laboratory testing for 2019 novel coronavirus (2019-nCoV) in suspected human cases: https://www.who.int/publications/i/item/10665-331501 (Interim guidance 17th January 2020)]. All hospitalized patients received the hospital's standard-of-care therapy (applicable at the time of the study), consisting in hydroxychloroquine $400 \mathrm{mg} / 24 \mathrm{~h}$ first day and $200 \mathrm{mg} / 24 \mathrm{~h} 4$ days with azithromycin $500 \mathrm{mg} / 24 \mathrm{~h} 3$ days, plus ceftriaxone 1 or $2 \mathrm{~g} / 24 \mathrm{~h} 7$ days when there was bacterial superinfection. Patients with severe or critical conditions of pulmonary inflammation or clinical 
suspicion of cytokine storm were additionally treated with dexamethasone bolus ( $20 \mathrm{mg} / \mathrm{day} \times 4$ days) according to hospital guidelines.

SARS-CoV-2 patients were placed in one of the 8 COVID-19 wards according to bed availability at the admission time. All COVID-19 wards followed the same hospital's standard of care protocol for COVID-19 treatment except for calcifediol, which was prescribed to patients in 5 wards whereas no such therapy was given to patients in the other 3 wards. Oral calcifediol treatment [25OHD3] (Hidroferol@ Faes-Farma, Lejona, Spain), in soft capsules was administered as following: first dose of 2 capsules (266 micrograms/capsule) at baseline (day 0), a second dose of 1 capsule at day 3 , and subsequent doses of 1 capsule given at days 7,15 , and 30 . The non-treated control group were patients who did not receive calcifediol at the time of hospitalization.

\section{Outcomes}

The outcomes of the study were admission into ICU (yes/no) and mortality (yes/no). The effect of calcifediol administration was studied in a prospective open label study. The consequences of baseline vitamin $D$ status were studied in a subset of 678 patients for whom baseline serum $250 H D$ concentrations were measured.

\section{Variables}

\section{Serum 25OHD levels}

Serum levels of $25 \mathrm{OHD}$ were measured at baseline through competitive immunoluminometric direct assay with direct-coated magnetic microparticles (coefficient of variation $<10 \%$ ) (Elecsys 25OHD total II, model 07028148190; Cobas e801 system, Roche Diagnostics GmbH, Mannheim, Germany). Obtained baseline levels were linearized using the decimal logarithm (logarithm with base 10 or $\log 10)$ to fit the multivariate regression analysis. Moreover, participants were classified according their $25 \mathrm{OHD}$ levels: patients with a serum concentration below $20 \mathrm{ng} / \mathrm{ml}$ were considered to be vitamin $D$ deficient, while those with $\geq 20 \mathrm{ng} / \mathrm{mL}$ were considered to be vitamin $\mathrm{D}$ replete $(17,18)$. 


\section{$\underline{\text { Risk factors and comorbidities }}$}

At the time of recruitment, several clinical variables and risk factors were registered from our hospital's and/or primary care medical records including: age, gender, high blood pressure (HBP), obesity (body mass index $\geq 30$ ), dyslipidemia, cardiovascular disease (CVD) including ischemic heart disease, congestive heart failure, arrythmia, heart valve disorders, and stroke, chronic kidney disease (CKD), type 1 or type 2 diabetes mellitus, respiratory-related diseases including chronic obstructive pulmonary disease and asthma, previous or current cancer, persistent viral infections including HIV, $\mathrm{HCV}$, and HBV, and autoimmune diseases including lupus, psoriasis, and rheumatoid arthritis.

\section{Statistical methods}

Descriptive statistics were used for demographic, laboratory, and clinical prognostic factors related to COVID-19. Comparisons between groups for quantitative variables were performed by Intention to treat analysis using t-test or Kruskal-Wallis test. Chi-square tests were used for qualitative variables and crosstabs to estimate the Odds Ratio (OR). Multivariate logistic regressions were used to estimate adjusted OR or Beta-coefficient ( $\beta$-coef), and $95 \%$ Cls for the probability of admission to ICU or mortality. Baseline levels of $25 \mathrm{OHD}$ were linearized by applying the base 10 logarithm. Age, sex and COVID-19 risk factors (i.e. HBP, dyslipidemia, CVD, obesity, previous or current cancer, CKD, chronic infections, autoimmune conditions, chronic respiratory diseases and type II diabetes mellitus) were selected as confounders. Statistical analysis was done with R for Windows version 3.5.3 (haven, stats, gam and compareGroups packages) and SPSS Statistics version 22.0. P values lower than 0.05 were considered significant.

\section{Results}

\section{Patient characteristics}

A total of 832 participants were included in the intention-to-treat analysis (ITT analysis). Of them, 447 patients were treated with calcifediol at admission and 391 were not treated. Fifty-three patients of this non-treated group started calcifediol during ICU admission. These patients were allocated in the treated group in a second analysis (Figure 1). Patient characteristics, stratified by calcifediol treatment at admission (ITT) are reported in Table 1A. Significant differences were found in baseline 25OHD 
levels between groups where the treated group had a median higher than the non-treated group (13 [8;24] vs.12 [8;19], respectively; $p<0.026)$. No other significant baseline differences were found in demographic characteristics, features and illness condition between groups.

A total of 678 patients in the cohort had baseline 25OHD levels measured, with a median [Q1;Q3] of 13 [8;22] $\mathrm{ng} / \mathrm{mL}$. Of them, $478(70.5 \%)$ had $25 \mathrm{OHD}$ levels $<20 \mathrm{ng} / \mathrm{ml}: 270(56.4 \%)$ in the treated group and $208(43.5 \%)$ in the untreated group.

Effects of calcifediol treatment and baseline $250 \mathrm{HD}$ levels on ICU admission.

ICU assistance was required by 102 participants (12.2\%). Out of 447 patients treated with calcifediol (at admission), $20(4.5 \%)$ required ICU, while out of 391 patients non-treated with calcifediol at admission, $82(21.0 \%)$ required ICU (OR: 0.18 [0.11;0.29, p value<0.0001). Patients admitted to the ICU had significantly lower baseline 25OHD levels compared to patients who remained in COVID wards (median [Q1;Q3]: ICU patients 10 [7;14]; No-ICU 13 [8;23] ng/ml, p value<0.0001). Obesity was significantly more frequent in ICU patients (Table 1B). Logistic regression of calcifediol treatment on ICU admission, adjusted by age, gender, linearized 25OHD levels at baseline, and comorbidities showed that treated patients had an $87 \%$ reduced risk to require ICU (OR 0.13 [95\% Cl $0.07 ; 0.23]$ ) (Table 2). Baseline 25OHD levels inversely correlated with the risk of ICU admission ( $\beta$-coef -1.72 [95\% Cl $-2.78 ;-0.71] ; p=0.001$ ). The same type of analysis was performed using categorized 25OHD levels ( $<20 \mathrm{ng} / \mathrm{ml}$ or $\geq 20 \mathrm{ng} / \mathrm{ml}$ ) showing that patients with adequate baseline levels ( $\geq 20 \mathrm{ng} / \mathrm{ml}$ ) had a decreased risk of ICU admission of 70\% compared to patients with deficient $25 \mathrm{OHD}$ levels (OR 0.30 [95\% Cl 0.14;0.65]) (Table 2). Obesity was also significantly associated with ICU admission (OR 2.34 [95\% Cl 1.10;4.93]) (Table 2). 
Effects of calcifediol treatment and baseline $25 \mathrm{OHD}$ levels on mortality

Out of 838 hospitalized patients included into the ITT analysis, 83 died (10\%). Of them, $31.3 \%$ died during ICU admission. First, we calculated the mortality risk according to the initial ITT: out of 447 patients who received calcifediol at admission, 21 died (4.7\%), whereas of the control group $(n=391)$, $62(15.9 \%)$ died (OR: 0.26 [0.15;0.43], p<0.0001). Logistic regression analysis adjusted by $25 \mathrm{OHD}$ levels, age, gender, and comorbidities showed that calcifediol treatment reduced the mortality risk by more than $70 \%(\mathrm{OR} 0.21[95 \% \mathrm{Cl} 0.10 ; 0.43])$ (Table 3). In a second analysis, the patients who received calcifediol at hospital admission $(n=447)$ were combined with the ones starting with this drug when admitted to the ICU ( $n=53)$ : of these 500 patients, 36 died $(7.2 \%)$ versus 47 deaths $(13.9 \%)$ in the 338 subjects who never received calcifediol (OR: 0.48 [0.30;0.76], $p=0.001$ ). Logistic regression analysis adjusted by 25OHD levels, age, gender, and comorbidities showed a reduction of mortality risk in treated individuals (OR $0.52[95 \% \mathrm{Cl} 0.27 ; 0.99]$ ) (Table 4). An additional analysis was performed excluding those 53 patients who started calcifediol treatment during ICU admission: Out of 447 patients treated with calcifediol, 21 (4.7\%) died, while out of 338 patients of the non-treatment group, 47 (13.9\%) died (OR: 0.30 [0.18;0.52], p value<0.0001). Logistic regression analysis adjusted by $25 \mathrm{OHD}$ levels, age, gender, and comorbidities showed that calcifediol treatment was associated with decreased mortality (OR $0.28[95 \% \mathrm{Cl} 0.13 ; 0.60]$ ) compared to non-treated patients (Table 5). COVID-19 patients who died had lower baseline 25OHD levels compared to patients who survived (median [Q1;Q3]: exitus patients $9 \mathrm{ng} / \mathrm{ml}$ [6;13.5]; discharged alive patients $13 \mathrm{ng} / \mathrm{ml}$ [8;22.7], $\mathrm{p}$ value $<0.0001$ ). Adjusted results showed that baseline $25 \mathrm{OHD}$ levels were significantly associated with mortality where adequate $25 \mathrm{OHD}$ levels reduced the mortality risk about $70 \%$ (Table 3 and 4 ). Linear 25OHD levels at baseline inversely correlated with mortality with a $\beta$-coef $-2.32[95 \% \mathrm{Cl}-3.77$; 0.99] $(p<0.001)$. Age, autoimmune disorders, and obesity were identified as risk factors for mortality (Tables 3 and 4).

As a subanalysis, we evaluated the mortality of ICU admitted patients $(n=102)$ : The mortality rate of such patients who received calcifediol at time of hospitalization $(n=20)$ was $10.0 \%$ versus $28.3 \%$ of mortality in subjects who started calcifediol after ICU admission $(n=53)$ and $31 \%$ in patients never treated with calcifediol $(n=29)$. No statistically significant differences were found between groups. 


\section{DISCUSSION}

In this open label study conducted during the first European outbreak of the deadly COVID-19 pandemic, we have observed that, in hospitalized COVID-19 patients, treatment with calcifediol reduced the risk of requirement for critical care by more than $80 \%$. This supports the conclusion of a prior pilot trial in Cordoba (Spain) in which calcifediol treatment led to a dramatic decrease ICU admission (odds ratio $0.03,95 \% \mathrm{Cl}: 0.003-0.25)(19)$. Furthermore, calcifediol started at the time of hospitalization (ITT analysis) reduced the mortality risk by more than $70 \%$. Importantly, our results suggest that calcifediol administration should preferably be given prior to ARDS development, since initiation of calcifediol during ICU admission did not modify patient survival to the same extent.

Studies with cholecalciferol have generated mixed results. In a rather underpowered Brazilian randomized clinical trial (20), a bolus of cholecalciferol $(200,000 \mathrm{IU}$ or $5 \mathrm{mg}$ of vitamin D3) administered during hospitalization because of COVID-19, did not reduce ICU admission nor the risk of death. In a small-scale study in hospitalized frail elderly COVID-19 patients, the administration of an oral supplement of $80,000 \mathrm{IU}$ vitamin D3 within hours of diagnosis also did not decrease the risk of death (21). However, vitamin D3 supplementation in bolus of 80,000 IU vitamin D3 either during the month before or in the week following diagnosis of COVID-19,_was associated with less severe COVID-19 evolution and better survival rate in frail elderly subjects (21). In addition, regular vitamin D3 supplementation, at least in the elderly, in boluses administered regularly during the year prior to diagnosis has been shown to reduce the risk of death and improve clinical outcome in elderly patients with COVID-19 $(22,23)$. In our study, patients treated with calcifediol previously to hospitalization were excluded for the ITT analysis since the main objective of this study was to analyze the effect of calcifediol administered at admission time.

Calcifediol, or 25OHD3, pre-hormone of the VDES, substrate for the synthesis of 1,25(OH)2D was selected, rather than more commonly used cholecalciferol or, native vitamin D3 threshold nutrient of the system, because of its excellent pharmacokinetic profile, including a high intestinal absorption (close to $100 \%$ ), a Tmax of approximately 4 hours resulting in a rapid increase in serum 25OHD, and a half-life of 12 - 22 days (24). The $\mathrm{AUC}_{0-72 \mathrm{~h}}(\mathrm{ng} / \mathrm{ml} \cdot \mathrm{h})$ is $2382.02 \pm 665.43(25)$. Moreover, based on 
all published comparative RCTs, oral calcifediol is more potent than oral cholecalciferol. When comparing a daily dose of less than $25 \mu \mathrm{g}$ of cholecalciferol (1000 IU) with similar low dosages of calcifediol, oral calcifediol is $2-5$-fold more potent (mean of all studies is about 3.2 ) than oral cholecalciferol, although the potency of calcifediol is much higher in studies using high-dose oral of cholecalciferol, varying from 5.5 to 9-12 times (24). Calcifediol does not require hepatic hydroxylation, which is frequently impaired in acutely ill patients, and thus more readably available for conversion to vitamin's D active metabolite, 1,25(OH)2D (24).

The study population had a relatively low vitamin D status as demonstrated by the median [Q1;Q3] serum $25 \mathrm{OHD}$ concentration at baseline $(13 \mathrm{ng} / \mathrm{mL}[8 ; 22])$. Whether the beneficial effects of calcifediol would also be applicable in less vitamin D deficient populations will require appropriate intervention studies.

The analysis of the Barna-COVIDIOL prospective cohort also showed that baseline serum 25OHD was significantly and inversely related to ICU requirement and mortality. Several observational studies have pointed to a relationship between vitamin D deficiency and COVID-19-related mortality and/or disease severity (26-29), including a large cross-sectional study performed across 20 European countries in which a negative correlation between $25 \mathrm{OHD}$ serum levels and mortality was observed (8). Panagiotou et al. also reported a significantly greater prevalence of vitamin D deficiency in COVID-19 patients admitted to the ICU compared to those that not required critical care (30). However, other studies failed to find an association between vitamin D deficient status and poor disease outcomes (30-33). Furthermore, we should take into consideration that lower 25(OH)D levels at baseline might reflect underlying comorbidities or an inflammatory state due to COVID-19 since $25(\mathrm{OH}) \mathrm{D}$ levels may decrease by up to $40 \%$ in states of systemic inflammation (34)(35). Therefore, it is plausible that patients with deficient Vitamin $D$ levels secondary to an inflammatory state are more likely to be admitted to the ICU. Recent publications have reviewed vitamin's D plausible immunomodulatory mechanisms of actions on SARS-CoV-2 infection which are likely to contribute to our study results $(8,19)$. Most likely, calcifediol interferes with COVID-19-induced ARDS development. Indeed, ARDS is the most common indication for admitting a patient with COVID-19 into the ICU (34). This life-threatening condition is the consequence of an inflammatory and diffuse alveolar injury of acute onset, which leads to bilateral lung infiltration and severe hypoxemia. The pathogenesis of ARDS is closely linked to an exacerbated pro-inflammatory cytokine response of the 
host (35), and it is precisely in this setting where we speculate that vitamin D exerted its main beneficial effects. The adaptive immune system can be modulated by $1,25(\mathrm{OH})_{2} \mathrm{D}$, most importantly by modifying the phenotype of dendritic cells (responsible for antigen presentation to $T$ cells) leading to a decrease in pro-inflammatory $\mathrm{T}$ cells subtypes proliferation, while enhancing the production of regulatory $\mathrm{T}$ cells (36). Pro-inflammatory cytokine release in macrophages is also decreased by $1,25(\mathrm{OH})_{2} \mathrm{D}(37)$. Ultimately these effects are thought to curb the inflammatory cascade that leads to the cytokine and chemokine storm associated with the pathogenesis of ARDS.

There might be other mechanisms by which vitamin $D$ might protect against ARDS (38). It downregulates the renin-angiotensin system (RAS), a pro-inflammatory endocrine axis implicated in ARDS pathogenesis through the decrease of renin activity and by increasing angiotensin converting enzyme 2 (ACE2) and controlling the alteration of the coagulation cascade. Intra-alveolar fibrin clots are particularly typical in coronavirus-provoked ARDS, and in addition to its inhibitory effects on RAS, several in vitro studies also suggest that $1,25(\mathrm{OH})_{2} \mathrm{D}$ displays direct anti-thrombotic effects. Finally, vitamin $\mathrm{D}$ appears to also play a role in the maintenance and repair of the respiratory epithelium, thus, potentially helping in the prevention and resolution of acute lung injury.

The study also confirmed that age and obesity were additional risk factors. Indeed, both factors have been linked to COVID-19 severity and poor outcomes in multiple studies and at the same time are well-established risk factors for vitamin D deficiency (39). Our analysis also included a logistic regression analysis eliminating the potential confounding effect of age and obesity.

There are several limitations in the present study. First, it was not placebo controlled and no electronic/statistical randomization was performed and hence, it cannot be considered as a randomized controlled trial. However, since incoming patients were assigned to a ward based on bed availability and therefore, were not selected, we can consider the effect of calcifediol close to an open label clinical study performed in real life conditions. Second, statistical differences in baseline 25OHD levels were found between treated and non-treated patients in the ITT analysis, even though these differences cannot be considered clinically relevant. Of note, all analyses were adjusted by these $25 \mathrm{OHD}$ levels as well as other confounder variables. Another limitation is that serum 25OHD was not measured during follow-up. No dose response curve was tested, so that we cannot define the minimal required dose and there was also no comparison with the more commonly used forms of vitamin D. 
However, considering the administered doses and the drug's pharmacokinetic profile, we assume it replenished 25OHD deposits in all treated patients. Finally, severity scales were not analyzed.

In spite of its weaknesses, the study was adequately powered to detect possible effects on the essential hard end points of ICU admission and mortality.

In summary, calcifediol administered shortly after hospitalization markedly reduced the requirement for ICU admission and decreased mortality by more than $50 \%$. Moreover, baseline $25 \mathrm{OHD}$ levels negatively correlated with ICU admission and mortality. These findings point to the relevance of attaining an adequate 25OHD status as soon as possible in the setting of SARS-CoV2 infection. This is particularly attractive in the current epidemiologic situation as $25 \mathrm{OHD}$ deficiency is an easily modifiable factor. Nonetheless, additional studies are necessary to fully elucidate the effects of circulating 25OHD levels and 25OHD treatment on COVID-19 disease severity in other populations with different baseline vitamin D status. 
Data Availability

Some or all datasets generated during and/or analyzed during the current study are not publicly available but are available from the corresponding author on reasonable request.

\section{Acknowledgments}

The research was supported by Centro de Investigación Biomédica en Red de Fragilidad y Envejecimiento Saludable (grants number CB16/10/00245 and CB16/10/00501); Formación en Investigación en Salud grants (grant number PI19/00033) from Instituto de Salud Carlos III (ISCIII); and European Regional Development Fund and COVID-011-2020 Consejería de Salud y Familia. "Fundación Progreso y Salud" and "Fundación para la Investigación Biomédica de Córdoba" (FIBICO), Andalucía, Spain. DO is recipient of a Sara Borrell grant from ISCIII. The COVIDMar group is also acknowledged: Silvia Gómez-Zorrilla, Inmaculada López-Montesinos, Maria Luisa Sorli-Redó, Joan Gómez-Junyent, Eloy García-Vives, Emili Letang, Itziar Arrieta-Aldea, Maria Mar Arenas-Miras, Maria Milagros Montero, and Juan Pablo Horcajada. 
1. Guan WJ, Ni ZY, Hu Y, Liang WH, Ou CQ, He JX, et al. Clinical Characteristics of Coronavirus Disease 2019 in China. N Engl J Med. 2020;382(18):1708-20.

2. Boban M. Novel coronavirus disease (COVID-19) update on epidemiology, pathogenicity, clinical course and treatments. Int J Clin Pract. 2020:e13868.

3. Russo F, Pitter G, Da Re F, Tonon M, Avossa F, Bellio S FU, Gubian L, Monetti D, Saia M, Zanella F, Zorzi M, Narne E, Mantoan D. Epidemiology and public health response in early phase of COVID-19 pandemic, Veneto Region, Italy, 21 February to 2 April 2020. Euro Surveill 2020;25(47).

4. Beigel JH, Tomashek KM, Dodd LE, Mehta AK, Zingman BS, Kalil AC, et al. Remdesivir for the Treatment of Covid-19 - Final Report. N Engl J Med. 2020;383(19):1813-26.

5. Group RC, Horby P, Lim WS, Emberson JR, Mafham M, Bell JL, et al. Dexamethasone in Hospitalized Patients with Covid-19 - Preliminary Report. N Engl J Med. 2020.

6. Kalil AC, Patterson TF, Mehta AK, Tomashek KM, Wolfe CR, Ghazaryan V, et al. Baricitinib plus Remdesivir for Hospitalized Adults with Covid-19. N Engl J Med. 2020.

7. Nirula A, Shen L, Skovronsky DM. Neutralizing Antibody LY-CoV555 for Outpatient Covid-19. Reply. N Engl J Med. 2020;384(2).

8. Ilie PC, Stefanescu S, Smith L. The role of vitamin D in the prevention of coronavirus disease 2019 infection and mortality. Aging Clin Exp Res. 2020;32(7):1195-8.

9. Meltzer DO, Best TJ, Zhang H, Vokes T, Arora V, Solway J. Association of Vitamin D Status and Other Clinical Characteristics With COVID-19 Test Results. JAMA Netw Open. 2020;3(9):e2019722.

10. Bishop E, Ismailova A, Dimeloe SK, Hewison M, White JH. Vitamin D and immune regulation: antibacterial, antiviral, anti-inflammatory. JBMR Plus. 2020.

11. Brighenti S, Bergman P, Martineau AR. Vitamin D and tuberculosis: where next? J Intern Med. 2018.

12. Greiller CL, Suri R, Jolliffe DA, Kebadze T, Hirsman AG, Griffiths $C$, et al. Vitamin D attenuates rhinovirus-induced expression of intercellular adhesion molecule-1 (ICAM-1) and platelet-activating factor receptor (PAFR) in respiratory epithelial cells. J Steroid Biochem Mol Biol. 2019;187:152-9.

13. Martineau AR, Jolliffe DA, Hooper RL, Greenberg L, Aloia JF, Bergman P, et al. Vitamin D supplementation to prevent acute respiratory tract infections: systematic review and meta-analysis of individual participant data. BMJ. 2017;356:i6583.

14. Nair P, Venkatesh B, Center JR. Vitamin D deficiency and supplementation in critical illnessthe known knowns and known unknowns. Crit Care. 2018;22(1):276.

15. Grant WB, Lahore H, McDonnell SL, Baggerly CA, French CB, Aliano JL, et al. Evidence that Vitamin D Supplementation Could Reduce Risk of Influenza and COVID-19 Infections and Deaths. Nutrients. 2020;12(4).

16. Jolliffe DA, Camargo CA, Jr., Sluyter JD, Aglipay M, Aloia JF, Ganmaa D, et al. Vitamin D supplementation to prevent acute respiratory infections: a systematic review and meta-analysis of aggregate data from randomised controlled trials. Lancet Diabetes Endocrinol. 2021;9(5):276-92.

17. Bouillon R, Marcocci C, Carmeliet G, Bikle D, White JH, Dawson-Hughes B, et al. Skeletal and Extraskeletal Actions of Vitamin D: Current Evidence and Outstanding Questions. Endocr Rev. 2019;40(4):1109-51.

18. Amrein K, Scherkl M, Hoffmann M. 25(OH)D deficiency 2.0: an update on the current status worldwide. Eur J Clin Nutr. 2020;74:1498-513.

19. Entrenas Castillo M, Entrenas Costa LM, Vaquero Barrios JM, Alcala Diaz JF, Lopez Miranda J, Bouillon $\mathrm{R}$, et al. "Effect of calcifediol treatment and best available therapy versus best available therapy on intensive care unit admission and mortality among patients hospitalized for COVID-19: A pilot randomized clinical study". J Steroid Biochem Mol Biol. 2020;203:105751.

20. Murai IH, Fernandes AL, Sales LP, Pinto AJ, Goessler KF, Duran CSC, et al. Effect of a Single High Dose of Vitamin D3 on Hospital Length of Stay in Patients With Moderate to Severe COVID-19: A Randomized Clinical Trial. JAMA. 2021;325(11):1053-60. 
21. Annweiler C, Hanotte B, Grandin de l'Eprevier C, Sabatier JM, Lafaie L, Celarier T. Vitamin D and survival in COVID-19 patients: A quasi-experimental study. J Steroid Biochem Mol Biol. 2020;204:105771.

22. Cangiano B, Fatti LM, Danesi L, Gazzano G, Croci M, Vitale G, et al. Mortality in an Italian nursing home during COVID-19 pandemic: correlation with gender, age, ADL, vitamin D supplementation, and limitations of the diagnostic tests. Aging (Albany NY). 2020;12(24):24522-34.

23. Ling SF, Broad E, Murphy R, Pappachan JM, Pardesi-Newton S, Kong MF, et al. High-Dose Cholecalciferol Booster Therapy is Associated with a Reduced Risk of Mortality in Patients with COVID-19: A Cross-Sectional Multi-Centre Observational Study. Nutrients. 2020;12(12).

24. Quesada-Gomez JM, Bouillon R. Is calcifediol better than cholecalciferol for vitamin D supplementation? Osteoporos Int. 2018;29(8):1697-711.

25. Jetter A, Egli A, Dawson-Hughes B, Staehelin HB, Stoecklin E, Goessl R, et al. Pharmacokinetics of oral vitamin D(3) and calcifediol. Bone. 2014;59:14-9.

26. Carpagnano GE, Di Lecce V, Quaranta VN, Zito A, Buonamico E, Capozza E, et al. Vitamin D deficiency as a predictor of poor prognosis in patients with acute respiratory failure due to COVID19. J Endocrinol Invest. 2020.

27. Maghbooli Z, Sahraian MA, Ebrahimi M, Pazoki M, Kafan S, Tabriz HM, et al. Vitamin D sufficiency, a serum 25-hydroxyvitamin $D$ at least $30 \mathrm{ng} / \mathrm{mL}$ reduced risk for adverse clinical outcomes in patients with COVID-19 infection. PLoS One. 2020;15(9):e0239799.

28. Radujkovic A, Hippchen T, Tiwari-Heckler S, Dreher S, Boxberger M, Merle U. Vitamin D Deficiency and Outcome of COVID-19 Patients. Nutrients. 2020;12(9).

29. Ye K, Tang F, Liao X, Shaw BA, Deng M, Huang G, et al. Does Serum Vitamin D Level Affect COVID-19 Infection and Its Severity?-A Case-Control Study. J Am Coll Nutr. 2020:1-8.

30. Panagiotou G, Tee SA, Ihsan Y, Athar W, Marchitelli G, Kelly D, et al. Original publication: Low serum 25-hydroxyvitamin $\mathrm{D}(25[\mathrm{OH}] \mathrm{D})$ levels in patients hospitalized with COVID-19 are associated with greater disease severity. Clin Endocrinol (Oxf). 2020;93(5):629-30.

31. Baktash V, Hosack T, Patel N, Shah S, Kandiah P, Van Den Abbeele K, et al. Vitamin D status and outcomes for hospitalised older patients with COVID-19. Postgrad Med J. 2020.

32. Hastie CE, Pell JP, Sattar N. Vitamin D and COVID-19 infection and mortality in UK Biobank. Eur J Nutr. 2020.

33. Pizzini A, Aichner M, Sahanic S, Bohm A, Egger A, Hoermann G, et al. Impact of Vitamin D Deficiency on COVID-19-A Prospective Analysis from the CovILD Registry. Nutrients. 2020;12(9).

34. Xu Z, Shi L, Wang Y, Zhang J, Huang L, Zhang C, et al. Pathological findings of COVID-19 associated with acute respiratory distress syndrome. Lancet Respir Med. 2020;8(4):420-2.

35. Channappanavar R, Perlman S. Pathogenic human coronavirus infections: causes and consequences of cytokine storm and immunopathology. Semin Immunopathol. 2017;39(5):529-39.

36. Adorini L, Penna G. Dendritic cell tolerogenicity: a key mechanism in immunomodulation by vitamin D receptor agonists. Hum Immunol. 2009;70(5):345-52.

37. Helming L, Bose J, Ehrchen J, Schiebe S, Frahm T, Geffers R, et al. 1alpha,25Dihydroxyvitamin D3 is a potent suppressor of interferon gamma-mediated macrophage activation. Blood. 2005;106(13):4351-8.

38. Quesada-Gomez JM, Entrenas-Castillo M, Bouillon R. Vitamin D receptor stimulation to reduce acute respiratory distress syndrome (ARDS) in patients with coronavirus SARS-CoV-2 infections: Revised Ms SBMB 2020_166. J Steroid Biochem Mol Biol. 2020;202:105719.

39. Jordan RE, Adab P, Cheng KK. Covid-19: risk factors for severe disease and death. BMJ. 2020;368:m1198. 
Figure legends

Figure 1. Flowchart showing the number of patients recruited in the Barna-COVIDIOL cohort allocated according to calcifediol treatment at admission-Intention-to-Treat (ITT) or during hospitalization. *53 patients from control group who started calcifediol during ICU admission 
TABLES

Table 1A: Patient characteristics stratified according to calcifediol treatment at admission

\begin{tabular}{|lcc|c|}
\hline Calcifediol & $\begin{array}{c}\text { Treated } \\
\text { N=447 (53.3\%) }\end{array}$ & $\begin{array}{c}\text { Non-treated } \\
\text { N=391 (46.7\%) }\end{array}$ & P value \\
\hline Mean age (years) + SD & $61.81 \pm 15.5$ & $62.41 \pm 17.2$ & NS \\
\hline Gender (\% male) & $264(59.1 \%)$ & $231(59.1 \%)$ & NS \\
\hline $\begin{array}{l}\text { Baseline levels of 25OHD (median ng/mI } \\
\text { [Q1; Q3]) }\end{array}$ & $13[8 ; 24]$ & $12[8 ; 19]$ & 0.026 \\
\hline HBP (n(\%)) & $189(42.3 \%)$ & $182(46.5 \%)$ & NS \\
\hline Dyslipidemia (n(\%)) & $122(27.3 \%)$ & $120(30.7 \%)$ & NS \\
\hline CAD (n(\%)) & $75(16.8 \%)$ & $76(19.4 \%)$ & NS \\
\hline Obesity (n(\%)) & $35(9.0 \%)$ & $33(7.4 \%)$ & NS \\
\hline Cancer* (n(\%)) & $35(7.8 \%)$ & $35(9.0 \%)$ & NS \\
\hline Chronic kidney disease (n(\%)) & $29(6.5 \%)$ & NS \\
\hline Chronic infections (n(\%)) & $19(4.3 \%)$ & $16(4.1 \%)$ & NS \\
\hline Autoimmune conditions (n(\%)) & $11(2.5 \%)$ & NS \\
\hline Chronic respiratory diseases (n(\%)) & $69(15.4 \%)$ & NS \\
\hline Type II Diabetes Mellitus (n(\%)) & $93(20.8 \%)$ & $76(19.4 \%)$ & NS \\
\hline $\begin{array}{l}\text { Abbreviations: SD, standard deviation; Q, quartile; HBP, high blood pressure; CAD, cardiovascular } \\
\text { disease; NS, Non-significant. * previous cancer or current }\end{array}$ & & \\
\hline
\end{tabular}


Table 1B: Patient characteristics according to ICU requirements

\begin{tabular}{|c|c|c|c|}
\hline Patients with ICU requirement & $\begin{array}{l}\text { YES } \\
\mathrm{N}=102(12.2 \%)\end{array}$ & $\begin{array}{l}\text { NO } \\
\mathrm{N}=736(87.8 \%)\end{array}$ & $P$ value \\
\hline Mean age (years) $\pm(S D)$ & $61.20 \pm 14.7$ & $62.21 \pm 16.6$ & NS \\
\hline Gender (\% male) & $67(65.7 \%)$ & $428(58.2 \%)$ & NS \\
\hline $\begin{array}{l}\text { Baseline levels of } 25(\mathrm{OH}) \mathrm{D} \text { (median } \\
\mathrm{ng} / \mathrm{ml}[\mathrm{Q} 1 ; \mathrm{Q}] \text { ) }\end{array}$ & $10[7 ; 14]$ & $13[8 ; 23]$ & $<0.001$ \\
\hline HBP (n(\%)) & $49(48 \%)$ & $322(43.8 \%)$ & NS \\
\hline Dyslipidemia (n(\%)) & $25(24.5 \%)$ & $217(29.5 \%)$ & NS \\
\hline CAD (n(\%)) & $21(20.6 \%)$ & $130(17.7 \%)$ & NS \\
\hline Obesity (n(\%)) & $14(13.7 \%)$ & $54(7.3 \%)$ & 0.027 \\
\hline Cancer $^{*}(\mathrm{n}(\%))$ & $10(9.8 \%)$ & $60(8.2 \%)$ & NS \\
\hline Chronic kidney disease (n(\%)) & $3(2.9 \%)$ & $61(8.3 \%)$ & NS \\
\hline Chronic infections (n(\%)) & $3(2.9 \%)$ & $32(4.3 \%)$ & NS \\
\hline Autoimmune conditions (n(\%)) & $5(4.9 \%)$ & $17(2.3 \%)$ & NS \\
\hline Chronic respiratory diseases (n(\%)) & $19(18.6 \%)$ & $136(18.5 \%)$ & NS \\
\hline Type II Diabetes Mellitus (n(\%)) & $25(24.5 \%)$ & $144(19.6 \%)$ & NS \\
\hline
\end{tabular}


Table 2: Logistic regression analysis for the association of calcifediol treatment and 25OHD baseline serum levels with ICU requirement.

\begin{tabular}{|c|c|c|c|c|}
\hline \multicolumn{5}{|c|}{ Using linearized baseline 250HD levels ( $n=678)$} \\
\hline & OR & Low Cl & High Cl & $p$ value \\
\hline Calcifediol treatment & 0.13 & 0.07 & 0.23 & $<0.001$ \\
\hline Linear 25OHD & 0.18 & 0.06 & 0.50 & 0.001 \\
\hline Obesity & 2.34 & 1.10 & 4.93 & 0.026 \\
\hline \multicolumn{5}{|c|}{ Using categorized baseline 25OHD levels $(n=678)$} \\
\hline & OR & Low Cl & High Cl & $p$ value \\
\hline Calcifediol treatment & 0.13 & 0.07 & 0.24 & $<0.001$ \\
\hline $25 \mathrm{OHD}(>=20 \mathrm{ng} / \mathrm{mL})$ & 0.30 & 0.14 & 0.65 & 0.002 \\
\hline Obesity & 2.10 & 1.00 & 4.40 & 0.048 \\
\hline
\end{tabular}

Adjusted by age, gender, 25OHD levels, and COVID-19 risk factors. Abbreviations: $\mathrm{Cl}$. $95 \%$ confidence interval; Linear 25OHD. baseline values of 25OHD linearized by base 10 logarithm; Obesity. body mass index $\geq 30$; OR. Odd Ratio; Calcifediol treatment. minimum of 2 capsules before ICU admission. 
Table 3: Logistic regression analysis for the association of calcifediol treatment at admission and $250 H D$ baseline serum levels with mortality. Original ITT analysis.

\begin{tabular}{|c|c|c|c|c|}
\hline \multicolumn{5}{|c|}{ Using linearized baseline 25OHD levels ( $n=678)$} \\
\hline & OR & Low Cl & High Cl & $p$ value \\
\hline Calcifediol treatment & 0.21 & 0.10 & 0.43 & $<0.001$ \\
\hline Linear 25OHD & 0.09 & 0.02 & 0.39 & 0.001 \\
\hline Age & 1.11 & 1.07 & 1.15 & $<0.001$ \\
\hline Autoimmune conditions & 5.78 & 1.32 & 25.30 & 0.02 \\
\hline Obesity & 3.48 & 1.30 & 9.33 & 0.013 \\
\hline \multicolumn{5}{|c|}{ Using categorized baseline 25OHD levels $(n=678)$} \\
\hline & OR & Low Cl & High Cl & $p$ value \\
\hline Calcifediol treatment & 0.22 & 0.11 & 0.44 & $<0.001$ \\
\hline $25 \mathrm{OHD}(>=20 \mathrm{ng} / \mathrm{mL})$ & 0.30 & 0.11 & 0.78 & 0.015 \\
\hline Age & 1.11 & 1.08 & 1.15 & $<0.001$ \\
\hline Autoimmune conditions & 6.17 & 1.37 & 27.72 & 0.018 \\
\hline Obesity & 2.94 & 1.10 & 7.87 & 0.031 \\
\hline \multicolumn{5}{|c|}{$\begin{array}{l}\text { Adjusted by age, Gender, } 25 \mathrm{OHD} \text { levels and COVID-19 risk factors. Abbreviations: } \mathrm{Cl} \text {. } 95 \% \\
\text { confidence interval; Linear } 25 \mathrm{OHD} \text {. baseline values of } 25 \mathrm{OHD} \text { linearized by base } 10 \text { logarithm; } \\
\text { Obesity. body mass index } \geq 30 \text {; OR. Odd Ratio; Calcifediol treatment. minimum of } 2 \text { capsules before } \\
\text { ICU admission. }\end{array}$} \\
\hline
\end{tabular}


Table 4: Logistic regression analysis for the association of calcifediol and baseline 25OHD serum levels with mortality. Treated group: all patients treated during hospitalization; Non-treated group: patients never treated.

\begin{tabular}{|c|c|c|c|c|}
\hline \multicolumn{5}{|c|}{ Using linearized baseline 25OHD levels ( $n=678$ ) } \\
\hline & OR & Low Cl & High Cl & $p$ value \\
\hline Calcifediol treatment & 0.52 & 0.27 & 0.99 & 0.048 \\
\hline Linear 25OHD & 0.10 & 0.02 & 0.38 & 0.001 \\
\hline Age & 1.11 & 1.07 & 1.14 & $<0.001$ \\
\hline Autoimmune conditions & 7.01 & 1.58 & 31.13 & 0.010 \\
\hline Obesity & 4.42 & 1.74 & 11.21 & 0.002 \\
\hline \multicolumn{5}{|c|}{ Using categorized baseline 25OHD levels ( $n=678$ ) } \\
\hline & OR & Low Cl & High Cl & $p$ value \\
\hline Calcifediol treatment & 0.55 & 0.29 & 1.04 & 0.066 \\
\hline $25 \mathrm{OHD}(>=20 \mathrm{ng} / \mathrm{mL})$ & 0.29 & 0.11 & 0.74 & 0.010 \\
\hline Age & 1.11 & 1.07 & 1.15 & $<0.001$ \\
\hline Autoimmune conditions & 6.90 & 1.53 & 31.06 & 0.012 \\
\hline Obesity & 3.71 & 1.45 & 9.50 & 0.006 \\
\hline \multicolumn{5}{|c|}{$\begin{array}{l}\text { Adjusted by age, gender, } 25 \mathrm{OHD} \text { levels and COVID- } 19 \text { risk factors, Abbreviations: } \mathrm{Cl}, 95 \% \\
\text { confidence interval; Linear } 25 \mathrm{OHD} \text {, baseline values of } 25 \mathrm{OHD} \text { linearized by base } 10 \text { logarithm; } \\
\text { Obesity, body mass index } \geq 30 ; \text { OR, Odd Ratio; Calcifediol treatment minimum of } 2 \text { capsules. }\end{array}$} \\
\hline
\end{tabular}


Table 5: Logistic regression analysis for the association of calcifediol treatment at admission and baseline 25OHD levels with mortality. Patients treated during ICU admission were excluded from the analysis.

\begin{tabular}{|c|c|c|c|c|}
\hline \multicolumn{5}{|c|}{ Using linearized baseline 25OHD levels ( $n=631$ ) } \\
\hline & OR & Low Cl & High Cl & $p$ value \\
\hline Calcifediol treatment & 0.28 & 0.13 & 0.60 & 0.001 \\
\hline Linear 25OHD & 0.10 & 0.021 & 0.45 & 0.003 \\
\hline Age & 1.13 & 1.09 & 1.18 & $<0.001$ \\
\hline Autoimmune conditions & 6.14 & 1.08 & 34.89 & 0.041 \\
\hline \multicolumn{5}{|c|}{ Using categorized baseline 250HD levels ( $n=631$ ) } \\
\hline & OR & Low Cl & High Cl & $p$ value \\
\hline Calcifediol treatment & 0.29 & 0.14 & 0.62 & 0.001 \\
\hline $25 \mathrm{OHD}(>=20 \mathrm{ng} / \mathrm{mL})$ & 0.39 & 0.14 & 1.06 & 0.065 \\
\hline Age & 1.13 & 1.09 & 1.18 & $<0.001$ \\
\hline Autoimmune conditions & 5.81 & 1.01 & 33.18 & 0.048 \\
\hline \multicolumn{5}{|c|}{$\begin{array}{l}\text { Adjusted by age, gender, } 25 \mathrm{OHD} \text { levels and COVID-19 risk factors. Abbreviations: } \mathrm{Cl}, 95 \% \\
\text { confidence interval; Linear } 25 \mathrm{OHD} \text {, baseline values of } 25 \mathrm{OHD} \text { linearized by base } 10 \text { logarithm; } \\
\text { Obesity, body mass index } \geq 30 \text {; OR, Odd Ratio; Calcifediol treatment at admission. }\end{array}$} \\
\hline
\end{tabular}


Figure 1

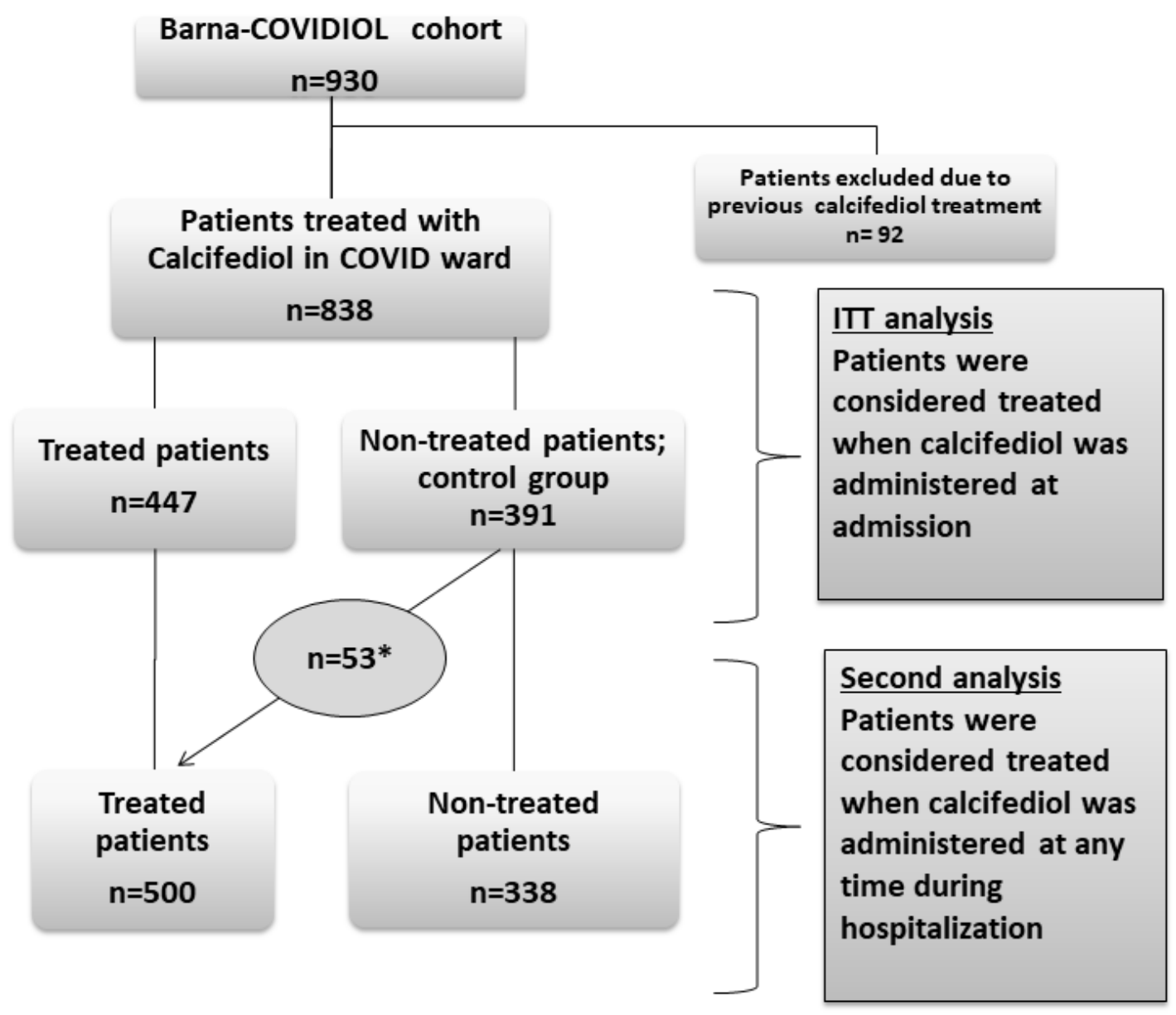

